\title{
The Significance of Amino Acid Inhibition of NADP-linked Glutamate Dehydrogenase in the Physiological Control of Glutamate Synthesis in Candida utilis*
}

\author{
By B. F. FOLKES AND A. P. SIMS \\ School of Biological Sciences, University of East Anglia, Norwich, NOR 88C
}

(Received 13 July 1973; revised 4 December 1973)

\begin{abstract}
SUMMAR Y
Isotopic nitrogen $\left({ }^{15} \mathrm{~N}\right)$ was used to measure the rate of synthesis of glutamic acid and of glutamine in turbidostat cultures of Candida utilis grown on ammonium phosphate as $\mathrm{N}$ source.

Steady-state experiments revealed that glutamic acid exists in more than one metabolic pool; approximately $5 \%$ of the free amino acid is in a separate 'storage' pool while a small but measurable component is apparently in a separate pool used specifically for the synthesis of glutamine. Over the temperature range 15 to $25^{\circ} \mathrm{C}$ the proportion of total nitrogen assimilated through glutamate was constant at $75 \% ; 12$ to $15 \%$ entered cellular metabolism through glutamine amide. Decrease of ammonium levels in the culture medium resulted in slightly lower growth rates and a decreased size of glutamate pool, but the proportion of nitrogen assimilated through glutamate remained constant.

Steady-state conditions could be disturbed by withholding nitrogen for a short period (Io to $20 \mathrm{~min}$ ) or by rapid shift of temperature. In both cases transient changes in growth rate, amino acid pools and rate of synthesis of glutamate and glutamine occurred. The rate of glutamate synthesis was very closely correlated with the size of the total pool of amino acids, and in vitro experiments confirmed that amino compounds were allosteric inhibitors of NADP-glutamate dehydrogenase. The in vivo data yielded a Hill number of 8 , indicative of the very sharp inhibition occurring over the range 0.15 to $0.25 \mathrm{M}$-amino acids. For extracted enzyme preparations the Hill number was smaller ( 2 to 6 ); this may imply partial dissociation at the lower protein concentrations used for in vitro experiments.

Analyses of organisms grown under steady-state conditions at various temperatures revealed that the amino acid feedback control on glutamate dehydrogenase is probably the chief mechanism involved in the regulation of glutamate synthesis to match growth requirements at different temperatures. Other physiological conditions in which glutamate formation is controlled are briefly discussed.
\end{abstract}

\section{INTRODUCTION}

In earlier studies on food yeast (Candida utilis) we showed from measurements of rates of incorporation of ${ }^{15} \mathrm{~N}$ into amino acids that the synthesis of glutamic acid was the only route in this organism by which amino compounds were formed from inorganic nitrogen (Sims \& Folkes, 1964). Furthermore, the kinetics of labelling of glutamic acid revealed that the enzymic reactions involved were not freely reversible within the cellular environment, since any appreciable rate of back reaction would have led to rates of isotope incorporation far in excess of those observed. The measured rates of labelling of most amino acids indicated rates of synthesis very close to those calculated to be necessary to sustain the observed rate

\footnotetext{
* We dedicate this paper to Professor E. W. Yemm, who retires in 1974.
} 
of growth, and this suggested that many of the synthetic reactions involved must be subject to metabolic control.

Glutamate synthesis in fungi is now generally believed to be by the NADP-linked glutamate dehydrogenase, the NAD-linked enzyme also found in such organisms being primarily a catabolic or deaminating system. Several reviewers have recently considered the regulation of these two enzymes in terms of the control of synthesis of enzymic protein in response to changes in level of possible inducers and repressors (Casselton, 1969; Shapiro \& Stadtman, 1970), but there is much less information about the effect of activators or inhibitors on the catalytic activity of these enzymes. The extensive studies of Barratt and of Fincham have revealed much about the structure and in vitro activation of the NADP. linked enzyme of Neurospora crassa (see, for example, West, Tuveson, Barratt \& Fincham, 1967), but there is still some uncertainty about the interpretation of these results since there is no guarantee that such observations need be relevant within the milieu of the living cell. It is for this reason that our study of the regulation of glutamate synthesis was confined almost exclusively to experiments in which the actual rates of reaction were measured in vivo. We attempted to identify effector ligands by looking for correlations between metabolic activity and the level of intermediates and end products within the cell, using a wide range of environmental conditions to minimize the chances of coincidence. The results, some of which have been briefly described elsewhere (Sims, Folkes \& Bussey, 1968), indicated that in Candida utilis the major control of glutamate synthesis rests with the cellular concentration of the free amino acids, themselves the end product of amino synthesis initiated by the formation of glutamate from ammonia.

\section{METHODS}

Organism. The organism used was Candida utilis NCYC737; this culture is derived from a single cell isolate from the strain BP60 used in earlier studies by Yemm \& Folkes (I954) and Sims \& Folkes (1964).

Cultural methods. All our experiments were carried out with organisms kept in continuous division in a turbidostat. Several different variants of this instrument were developed during the period covered by this work, so their general features only will be described.

In early models glass culture vessels were used. These were pyriform or cylindrical and held approximately 21 culture. Aeration and stirring were by means of air forced through a large sinter ( $10 \mathrm{~cm}$ diam, of glass or Teflon) set in the base of the vessel; air flows of 600 to $1500 \mathrm{ml} / \mathrm{min}$ were used, the air being previously brought to a controlled temperature and water saturation. More recently a Porton type 21 fermenter (Taylor Rustless Co. Ltd, Leeds 12) has been used, with both controlled air inflow and mechanical stirring. Essentially similar results were obtained with both types of system. With earlier models temperature was controlled by immersing the vessel in a constant temperature water bath fitted with optional refrigeration; in later models cold water from a refrigerated bath was circulated through cooling coils within the vessel and the temperature was raised to the desired point by an electric heater immersed in the culture. The turbidity of the culture was monitored by means of an optical cell mounted within either a twin beam or a split beam colorimeter. A peristaltic pump pumped the cell suspension from the culture vessel, through the optical cell and back to the culture vessel, and a degassing device was included in the pumpline to remove air bubbles before they could pass through the optical cell. As soon as the culture opacity exceeded a pre-set standard by $0.2 \%$ a system of electronic and electromagnetic controls allowed a measured volume (usually $5 \mathrm{ml}$ ) of fresh culture fluid to flow under gravity into 
the culture vessel and at the same time the event was recorded on a pen trace and on an event counter. Culture volume was kept constant by means of a fixed weir; alternatively a suck-out tube was held at a fixed level in the culture vessel and permanently connected to a peristaltic pumpline running to a waste sink.

By means of this apparatus it was possible to keep organisms in continuous culture for up to 3 weeks. During this period small changes of extinction occurred, partly caused by electronic instability and partly by a slow build-up of yeast cells on the face of the optical cell (this latter could be minimized by periodic cleaning). Over periods of a few hours variability of extinction was less than $0.5 \%$. Under steady-state conditions additions of fresh medium were made by the apparatus at intervals of less than a minute, and the regularity of the input trace gave clear evidence of the precision of the regulation. This precision made it possible to use the input trace for the calculation of changes in rates of growth and nutrient uptake during experiments in which transient changes in environmental conditions resulted in periods of non-steady-state growth and metabolism.

Analysis of yeast amino acids and estimation of ${ }^{15} \mathrm{~N}$-labelling. Samples (100 to $350 \mathrm{ml}$ ) of yeast removed directly from the turbidostat were filtered on glass-fibre filter paper and treated with $60 \%$ ethanol to extract amino acids; the extract was used for the colorimetric estimation of total amino acids and for the chromatographic separation of glutamic acid and glutamine prior to estimation of ${ }^{15} \mathrm{~N}$-labelling by mass spectrometry. All these methods were described by Sims \& Ferguson (1974).

Calculation of rates of synthesis from isotopic labelling. For steady-state experiments, use was made of the integrated labelling-rate equations derived by Sims \& Folkes (1964), but the experimental procedure was modified because the present experiments were carried out in a turbidostat. This instrument makes it much more certain that the experimental organisms are in steady-state conditions, but also raises the problem of how to bring about isotopic labelling of the ammonia in the culture vessel without disturbing the steady state. We overcame this difficulty by switching, at the start of the experiment, from an inflow medium containing unlabelled ammonium phosphate to one of exactly the same composition and concentration, except that the ammonium phosphate was labelled with ${ }^{15} \mathrm{~N}$. This input medium entered the culture vessel in response to cellular growth and there mixed with and labelled the residual ammonia of the culture. The form of the labelling curve with time was comparable with that for a primary amino acid as described by Sims \& Folkes (1964), the turnover number for the labelling being given by the ratio of the concentration of ammonia in the input medium to that of the residual ammonia in the culture. Cellular glutamic acid and glutamine then had labelling curves typical of secondary amino acids; their turnover constants could thus be evaluated as described for such amino acids by Sims \& Folkes (1964), assuming the turnover constant of their precursor pool to be that calculated from the turnover number of the residual ammonia.

For non-steady-state experiments it was not possible to apply purely numerical analysis as with the integrated rate equations. Instead, we used a graphical method based on the differential form of these equations. The underlying argument is as follows: Consider a reaction sequence

$$
A \underset{k_{1}}{\longrightarrow} B \underset{k_{2}}{\longrightarrow}
$$

in which $k_{1}$ and $k_{2}$ are transfer coefficients for the synthesis and utilization of $B$. Then in a quantity of yeast, $Q$,

$$
\frac{\mathrm{d} B}{\mathrm{~d} t}=k_{1} Q-k_{2} Q
$$


Now suppose that $A$ is labelled, the proportion of labelled molecules being $A^{+}=A^{*} \mid A$, where $A^{*}$ is the number of labelled molecules. $B$ will become labelled to $B^{+}$, and if the number of labelled molecules is $B^{*}$, then

From this it follows that

$$
\frac{\mathrm{d} B^{*}}{\mathrm{~d} t}=k_{1} Q A^{+}-k_{2} Q B^{+} \text {. }
$$

and hence

$$
\frac{\mathrm{d} B^{+}}{\mathrm{d} t}=\frac{\mathrm{d}\left(B^{*} / B\right)}{\mathrm{d} t}=k_{1} \frac{Q}{B}\left(A^{+}-B^{+}\right)
$$

$$
k_{1}=\frac{\mathrm{d} B^{+}}{\mathrm{d} t} \times \frac{B}{Q}\left(\frac{\mathrm{I}}{A^{+}-B^{+}}\right) .
$$

If measurements are made of $A^{+}, B^{+}$and $B / Q$ at various times, it is possible to estimate $\mathrm{d} B^{+} / \mathrm{d} t$ from tangents to the graph of $B^{+}$against time and hence to calculate $k_{1}$ (the rate of synthesis of $B$ in a unit quantity of yeast) at various times throughout the experiment.

Analytical procedures. Ammonia was measured in earlier experiments by the ascorbicninhydrin method used also for the estimation of amino acids; more recently the phenolhypochlorite method of Weatherburn (1967) has been used.

Protein was estimated using the method of Lowry, Rosebrough, Farr \& Randall (195I).

The method described here for RNA and DNA relies upon a simultaneous measurement of the free bases, uracil and thymine, obtained by acid hydrolysis of the yeast. Samples of ethanol-extracted yeast (about $25 \mathrm{mg}$ dry wt) were hydrolysed in $5 \mathrm{ml} 6 \mathrm{~N}-\mathrm{HCl}$ at $120^{\circ} \mathrm{C}$ for $2 \mathrm{~h}$ in sealed tubes. Each hydrolysate was filtered and evaporated to dryness and then applied in $3 \mathrm{ml}$ water to a column (IO $\times \mathrm{I} \mathrm{cm}$ ) of Dowex I (acetate) and washed in with a further $3 \mathrm{ml}$. The free bases were eluted from the column with $15 \mathrm{ml}$ water and then rechromatographed by applying, in I $\mathrm{ml}$, to a column $(20 \times \mathrm{I} \mathrm{cm})$ of Dowex $50\left(\mathrm{Na}^{+}\right)$maintained at $12{ }^{\circ} \mathrm{C}$ and previously equilibrated with sodium citrate buffer ( $0 \cdot 2 \mathrm{M}$-citric acid adjusted to $\mathrm{pH} 4.0$ with $\mathrm{NaOH}$ ). From this column the bases were eluted with water and collected in $\mathrm{I} .5 \mathrm{ml}$ fractions. Uracil and thymine emerged after about 20 and $35 \mathrm{ml}$ respectively and were measured at $260 \mathrm{~nm}$. The corresponding quantities of RNA and DNA were calculated by assuming uracil to represent $27 \%$ of the total bases in yeast RNA, and thymine $33 \%$ of the bases in yeast DNA (Belozersky \& Spirin, 1960).

Determination of organism number and size. Cell volume was measured with a Model B Coulter Counter (Coulter Electronics Ltd, Slough) fitted with a $70 \mu \mathrm{m}$ aperture. Counts were made on the same sample at various threshold settings and from these the relative cell size distribution was calculated in the standard way. Absolute size measurements were derived by carrying out a calibration of the instrument using as standards yeast samples whose mean cell size had been determined by microscopic measurement of Ioo individual cells using an eyepiece micrometer. Lengths and diameters of both buds and mother cells were measured and volumes calculated by assuming the cells to be cylindrical with hemispherical ends.

Enzymes. NADP-specific glutamate dehydrogenase [L-glutamate: NADP oxidoreductase (deaminating) (EC. I.4.I.4)] and glutamine synthetase [L-glutamate: ammonia ligase (ADP) (EC. 6.3.I.2)] were assayed by the methods of Ferguson \& Sims (197I). 

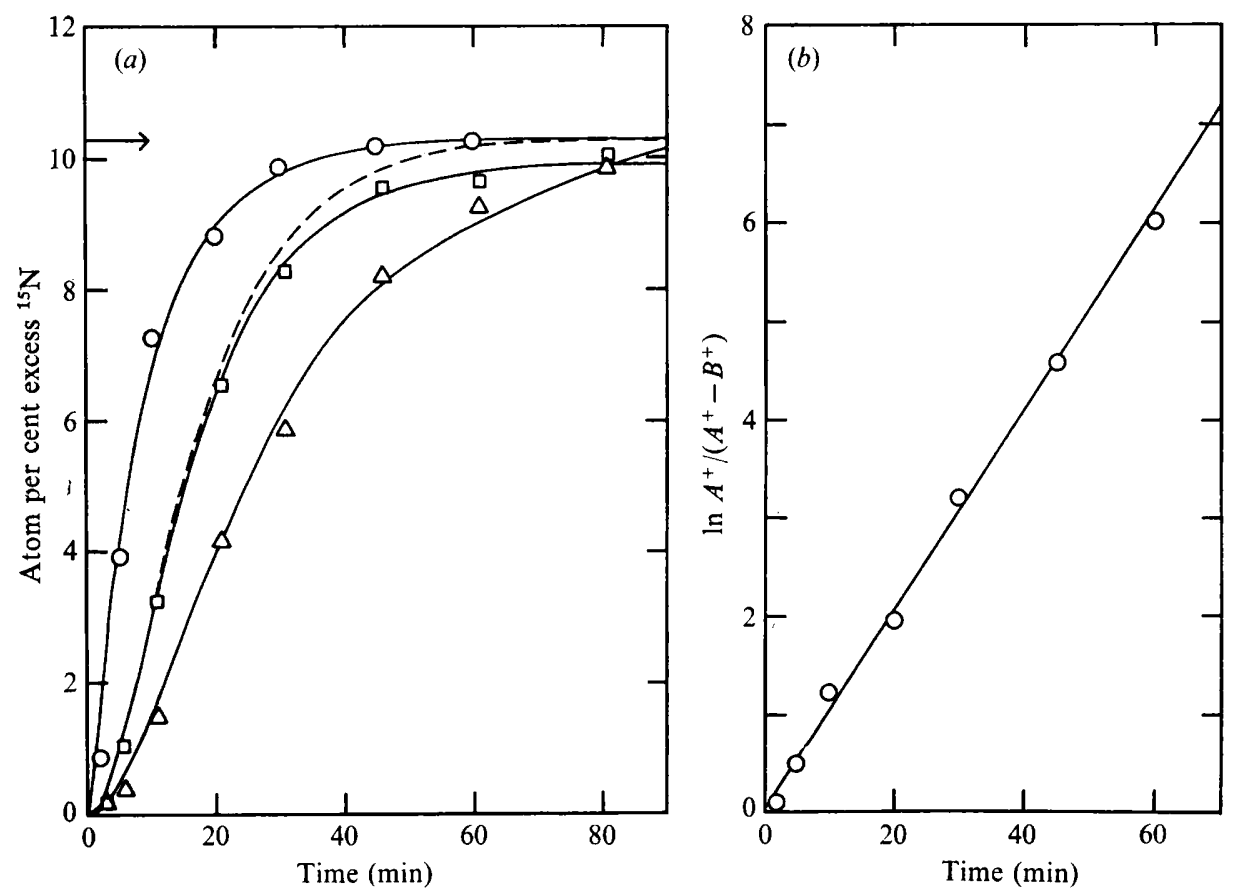

Fig. I. Steady-state isotope incorporation into a yeast culture grown in a turbidostat at $25^{\circ} \mathrm{C}$. The ammonia concentration in the culture vessel was maintained at $5.0 \mu \mathrm{g} \mathrm{N} / \mathrm{ml}$ and the specific growth rate or growth constant (a) was $0.006 \mathrm{I} 2 / \mathrm{min}$. The experimental procedure is described in Methods; the labelled input medium contained 10.28 atom per cent excess ${ }^{15} \mathrm{~N}\left(=A^{+}\right)$in the form of ammonium phosphate. $(a){ }^{15} \mathrm{~N}$-labelling in the ammonia of the culture vessel $(O)$, and in the free glutamic acid $(\square)$ and glutamine $(\triangle)$ of the yeast amino acid pool. The theoretical curves are as follows. Ammonia: Curve of $B^{+}=A^{+}\left(\mathrm{I}-\mathrm{e}^{-\mathrm{b} t}\right)$ where $\mathrm{b}=0.1028 / \mathrm{min}$, this value being derived from the best fit line in Fig. I $b$. Glutamate: The broken line is for

$$
C^{+}=A^{+}\left[\frac{\mathrm{c}\left(\mathrm{I}-\mathrm{e}^{-\mathrm{b} t}\right)-\mathrm{b}\left(\mathrm{I}-\mathrm{e}^{-\mathrm{c} t}\right)}{\mathrm{c}-\mathrm{b}}\right]
$$

where $\mathrm{c}=0.1 \mathrm{I} 4 / \mathrm{min}$. A better fit is given by the solid line, representing $95 \%$ of $C^{+}$and $5 \%$ of

$$
C_{\mathrm{p}}^{+}=A^{+}\left[\frac{\mathrm{bc}(\mathrm{b}-\mathrm{c})\left(\mathrm{I}-\mathrm{e}^{-\mathrm{a} t}\right)-\mathrm{ac}(\mathrm{a}-\mathrm{c})\left(\mathrm{I}-\mathrm{e}^{-\mathrm{b} t}\right)+\mathrm{ab}(\mathrm{a}-\mathrm{b})\left(\mathrm{I}-\mathrm{e}^{-\mathrm{ct}}\right)}{(\mathrm{b}-\mathrm{c})(\mathrm{a}-\mathrm{c})(\mathrm{a}-\mathrm{b})}\right],
$$

where $\mathrm{c}=0.120 / \mathrm{min}$. Glutamine: Curve of $C^{+}$where $\mathrm{c}=0.047 / \mathrm{min}$. (b) Plot of $\ln A^{+} /\left(A^{+}-B^{+}\right)$ for the observed values of $B^{+}$given by the isotopic labelling of the ammonia in culture vessel. The best fit straight line has a slope of $b=0 \cdot 1028 / \mathrm{min}$.

\section{RESULTS}

\section{Steady-state determinations of the rate of glutamate synthesis}

A typical steady-state experiment (Fig. I) revealed that the turbidostatic control of medium input was sufficiently good to give a labelling curve for medium ammonia which fitted closely that for a primary component. The results also largely confirmed earlier findings (Sims \& Folkes, I964) that the amino nitrogens of both glutamic acid and glutamine were directly derived from the ammonia in the medium and hence in the present experiment were fitted by secondary labelling curves. The more precise methods used here, including the much more truly steady-state growth conditions, made it possible however to detect minor deviations from the expected labelling curve which indicated that we were not dealing 
with a single simple metabolic pool but a mixture of at least two pools turning over at different rates. In the glutamate labelling this showed up most clearly towards the end of the experiment, where it was evident that the isotopic abundance levelled out at a slightly lower value than that reached by the ammonia in the medium. Thus a better fit to the data was given by a curve calculated on the basis of two pools, one a metabolic pool and the other a storage pool (Sims \& Folkes, I964), the latter constituting some $5 \%$ of the total. This, however, is only one possible model and it is not improbable that the second pool was larger than the assumed value and was turning over more rapidly than a true storage pool.

The earlier points on the glutamine graph (Fig. I) were consistently lower than the theoretical curve which accurately fitted the later points; this, together with recent observations by A. P. Sims \& A. R. Ferguson (unpublished) that the labelling of glutamine amide $N$ was always higher than that of glutamine amino $\mathrm{N}$, suggests that the precursor pool of glutamate used in glutamine synthesis may be of significant size (cf. Sims \& Folkes, 1964) or else that part of the glutamic acid used in glutamine synthesis may be derived from the breakdown of glutamine itself, as will occur when the latter is used as an amide donor in the synthesis of other compounds.

Clearly these complexities had a barely significant effect on our estimates of the overall rate of glutamate synthesis. Thus with the glutamate pool itself the total rate of synthesis deduced from the two-pool model was almost identical with that calculated for a single-pool model as long as only the earlier samples were considered; it was in the later samples, where labelling in glutamate approached that of the ammonia, that appreciable errors would arise from the use of a single-pool model. This point had been heeded in experiments involving non-steady-state conditions in which only the single-pool model could be applied; here care had been taken to ensure that the $A^{+}-B^{+}$term in the rate equation was kept large and under these conditions estimates of rate could not be in error by more than a few per cent.

The apparently direct derivation from ammonia of the amino group of glutamine suggested to Sims \& Folkes (1964) that the glutamyl of glutamine came from a source of newly synthesized glutamic acid which was not in equilibrium with the general glutamate pool. Estimates of the rate of glutamate synthesis based solely on the labelling of glutamate would thus be too small, because they would not include the glutamate going into glutamine. The complexities revealed in the present data do not materially affect this. Recycling of glutamyl residues may mean that the rate of synthesis of glutamine is underestimated from the labelling of its amino group, but this labelling will still give a reasonable estimate of the rate of flow of newly synthesized glutamate into glutamine. The slight lag in glutamine labelling due to the existence of a significant precursor pool will result in minor underestimates of rate, but the assessments of total glutamate synthesis cannot be more than I to $2 \%$ low.

Table I records the results of a number of steady-state experiments carried out under different conditions of temperature and ammonia concentration in the culture vessel. Ammonia concentration was controlled by altering the extinction of the culture at which fresh input of medium occurred; its concentration in the input medium was maintained constant at $84 \mu \mathrm{g} \mathrm{N} / \mathrm{ml}$ but at higher extinctions more of the ammonia was used up before additions occurred. In this way it was possible to decrease the ammonia concentration to a value at which its rate of uptake by the yeast was affected, resulting in changes in cell composition and growth rate of the type invariably associated with growth in a chemostat but not normally observed in a turbidostat. This phenomenon was demonstrated in Table I from a comparison of Experiments 2 and 3, the temperature difference here being too small to account for the observed differences in growth rate, glutamate level and total nitrogen content. Although the data for glutamine level were incomplete, this may have been particularly 
Table I. Steady-state rates of glutamate synthesis under varying conditions

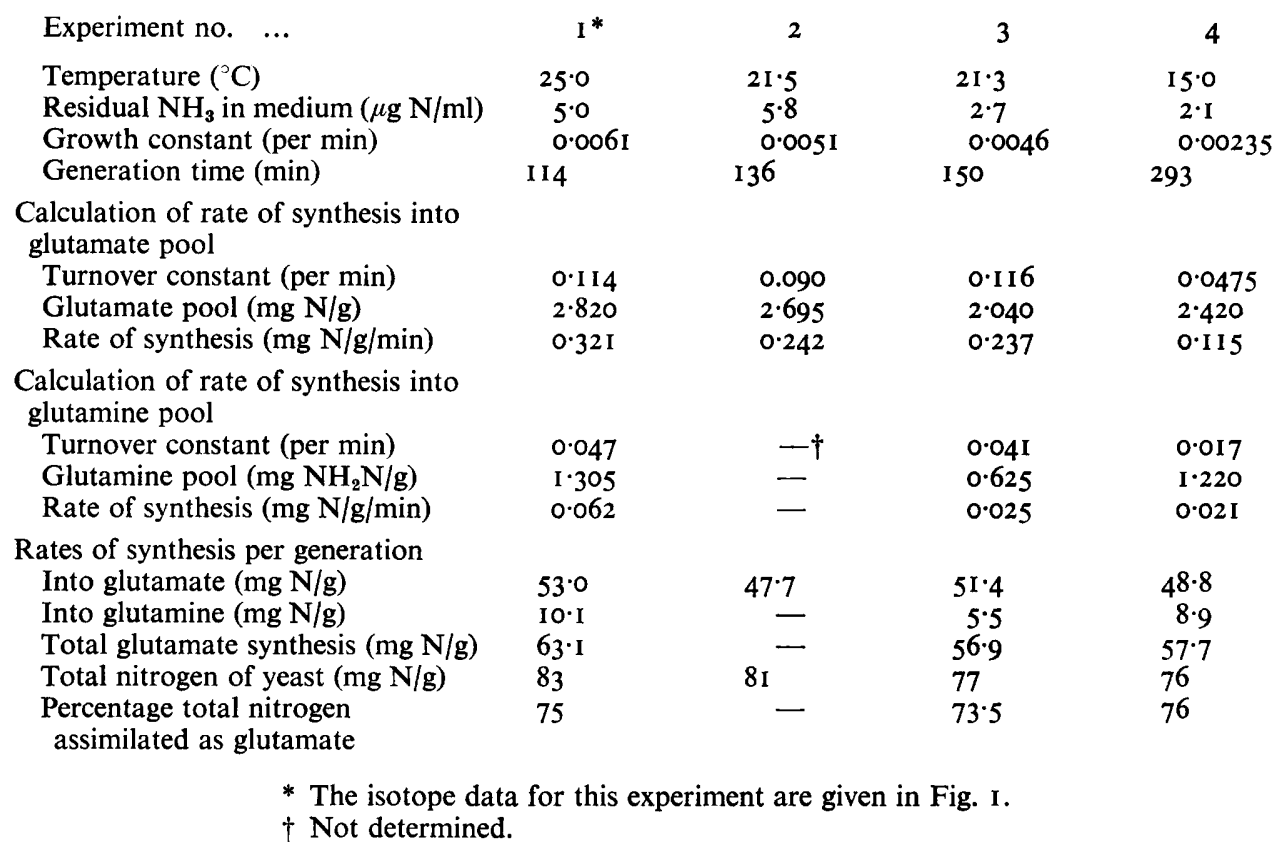

sensitive to nitrogen limitation (see Ferguson \& Sims, I974). Experiment 4 was probably also severely nitrogen limited, but at this temperature $\left(15{ }^{\circ} \mathrm{C}\right)$ the nucleic acid content was appreciably lower than at higher temperatures (see Fig. 5 below); it is likely therefore that there was a decreased demand for glutamine for nucleotide synthesis, which allowed the glutamine pool in this experiment to reach substantial levels despite the restricted supply of ammonia.

The rate of glutamate synthesis was remarkably uniform and, irrespective of temperature, was apparently regulated so that approximately $75 \%$ of the total nitrogen of the yeast was assimilated through glutamate. Assimilation through the amide group of glutamine probably accounted for at least a further $12 \%$, this estimate being minimal since it is based on amino labelling in glutamine. Nitrogen limitation did appear to decrease the rate of glutamate synthesis, but the lower growth rate and decreased nitrogen content of the yeast under these conditions meant that the decreased rate of synthesis was still adequate to meet growth requirements.

\section{Transient effects on the rate of glutamate synthesis following a brief period of nitrogen starvation}

Of several different ways to effect perturbations of the steady-state condition in order to follow the resulting changes in rate of glutamate synthesis, one of the most useful is to subject the yeast to a brief period of nitrogen starvation and then restore the supply of ammonia. Yeast was grown in the turbidostat on a medium containing $84 \mu \mathrm{g} \mathrm{N} / \mathrm{ml}$ and the density was adjusted so that the residual ammonia in the culture medium was kept at 4 to $5 \mu \mathrm{g} \mathrm{N} / \mathrm{ml}$. When steady-state growth was well established the inflow was switched to a new culture medium without ammonia, and the residual nitrogen then rapidly fell as assimilation continued. After a brief period (Io to $20 \mathrm{~min}$ depending on temperature) of complete 


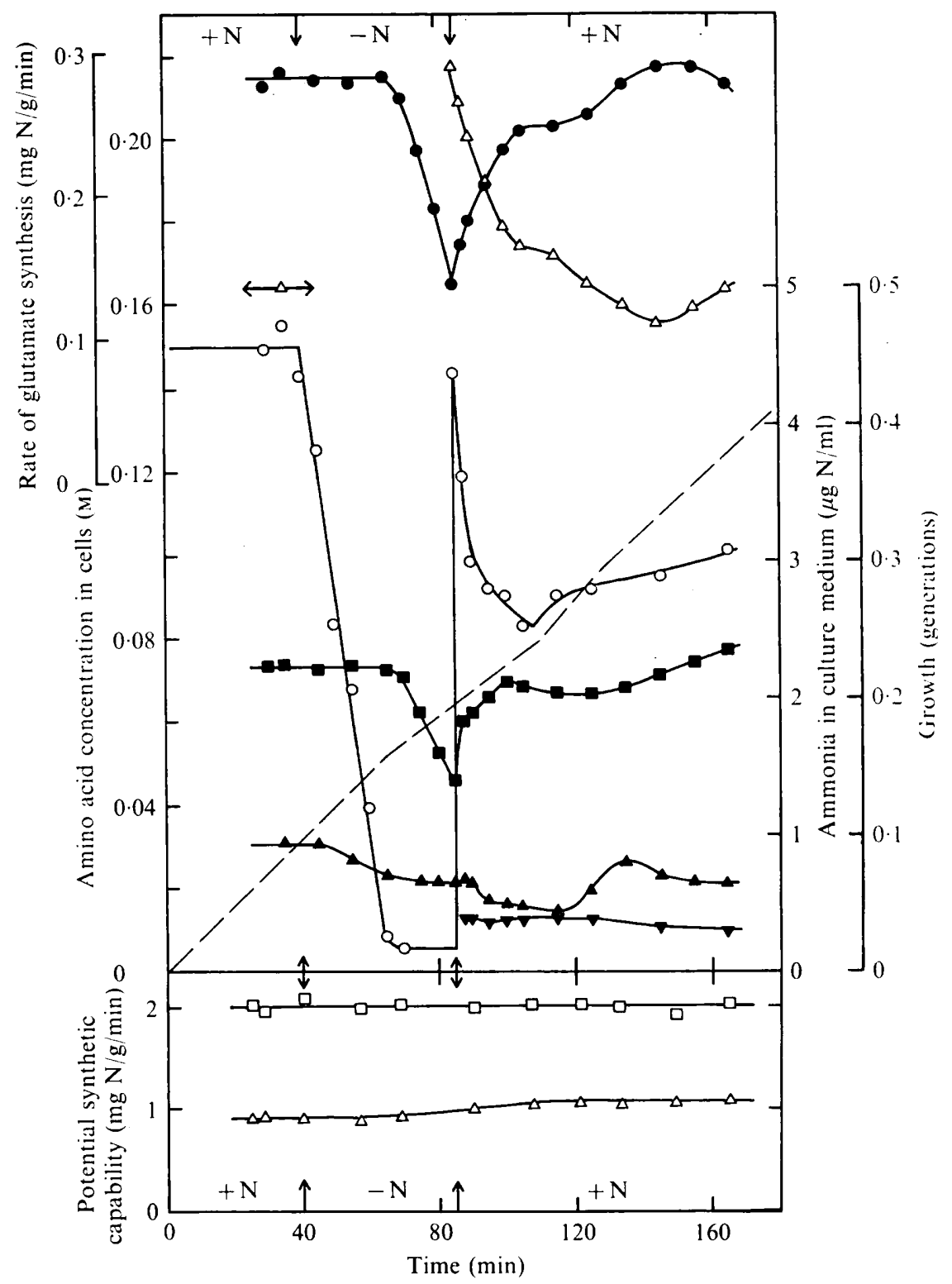

Fig. 2. The collected results of a number of nitrogen depletion experiments carried out at $15{ }^{\circ} \mathrm{C}$. The experimental procedure is described in the text. Upper graph: $\mathrm{O}$, ammonia in culture medium; $\boldsymbol{\theta}$, pool concentration of total amino acids; $\boldsymbol{\square}$, pool concentration of glutamic acid; $\boldsymbol{\Delta}$, pool concentration of glutamine; $\boldsymbol{\nabla}$, pool concentration of aspartic acid (data for complete time span not available); $\Delta$, rate of synthesis of glutamic acid after nitrogen restoration; $\leftarrow \Delta \rightarrow$, steady-state rate of synthesis of glutamic acid; - - yeast growth curve derived from the record of the time of addition of successive portions of fresh medium to the turbidostat (this is effectively a plot of addition number against time, so the slope indicates the growth rate). Lower graph: $\square$, intracellular level of NADP-glutamate dehydrogenase; $\triangle$, intracellular level (times 10 ) of glutamine synthesis. Both sets of assays are expressed in terms of the potential synthetic capability at $15{ }^{\circ} \mathrm{C}$ in the absence of feedback controls and substrate limitation. 
nitrogen starvation a small, calculated volume of ammonium phosphate solution was pipetted into the fermenter, the addition being just sufficient to restore the ammonium concentration to its original level. At the same time the inflow reverted to the nitrogen-containing medium. For isotope experiments this new inflow medium was labelled with $30 \%$ atom excess ${ }^{15} \mathrm{~N}$, while the ammonia addition was also labelled but to only $10 \%$ atom excess. It was thus possible to achieve throughout the experiment a progressive increase in the labelling of the ammonia of the culture which approximately matched the rate of labelling of the glutamate pool of the yeast. This ensured that the $A^{+}-B^{+}$term in the labelling equation never became small enough to invalidate the use of the single-pool model.

The results of several closely comparable experiments carried out at $15{ }^{\circ} \mathrm{C}$ are combined in Fig. 2. During the phase of nitrogen depletion, shown on the left of the graph, the residual ammonia level fell rapidly to a barely detectable value, and the yeast was then virtually deprived of nitrogen for a further $20 \mathrm{~min}$. As soon as the ammonia level began to fall the glutamine pool of the yeast started to decrease, at first rapidly but later more slowly, until it eventually stabilized at about $70 \%$ of its original value. In contrast, the pool.of glutamic acid and the total content of amino acids started to decline only when the ammonia in the medium was virtually exhausted, but then dropped rapidly. Addition of ammonia to the fermenter restored the ammonia in the culture to its previous concentration, but in spite of the inflow of new medium also containing ammonia the residual ammonia level rapidly fell, thus indicating a rate of ammonia uptake by the yeast which was far in excess of its steadystate value. Isotopic determinations of the rate of glutamate synthesis confirmed this, the initial rate being more than twice the steady-state value which it later approached and oscillated about in a characteristic manner.

These changes in rate of glutamate synthesis were clearly not causally connected with the changes in cell division rate. The latter decreased once the medium ammonia was exhausted, and did not increase again until the rate of glutamate synthesis had started to steady down; there was then a short burst of rapid growth before the cells settled into a rate of division comparable with the steady-state rate. Correlations with glutamate or glutamine level were also absent. The glutamate concentration did rise as the rate of synthesis fell, but the correspondence was poor; and in experiments in which the period of nitrogen depletion was shorter, the rate of glutamate synthesis followed a qualitatively similar curve, but glutamate concentration fell and instead the glutamine rose.

In all experiments, however, an extremely close correlation could be demonstrated between the rate of synthesis and the total pool of all amino acids, the curve for soluble $\alpha$-amino $\mathrm{N}$ almost exactly mirroring that for glutamate synthesis (Fig. 2). This observation suggested that amino acids generally could be involved in the mechanism of control. Measurements of enzyme level (Fig. 2) revealed that enzyme synthesis was not implicated: while assays of glutamine synthetase showed appreciable increases in synthetic capacity consequent upon the derepression associated with nitrogen starvation (Ferguson \& Sims, 1974), the content of the NADP-linked glutamate dehydrogenase remained virtually constant throughout the experiment, although following longer periods of nitrogen starvation extensive derepression of the synthesis of this enzyme also could be observed.

It thus seemed possible that the close correlation between the rate of glutamate synthesis and the level of $\alpha$-amino nitrogen (see also Fig. $3 a$ ) could be interpreted as an example of end-product feedback inhibition. Tests on the extracted NADP-linked glutamate dehydrogenase revealed that all the common amino acids were inhibitory, although to varying extents (Sims et al. I968). However, these differences between individual amino acids were not large so that, provided the relative composition of the amino acid pool remains fairly 

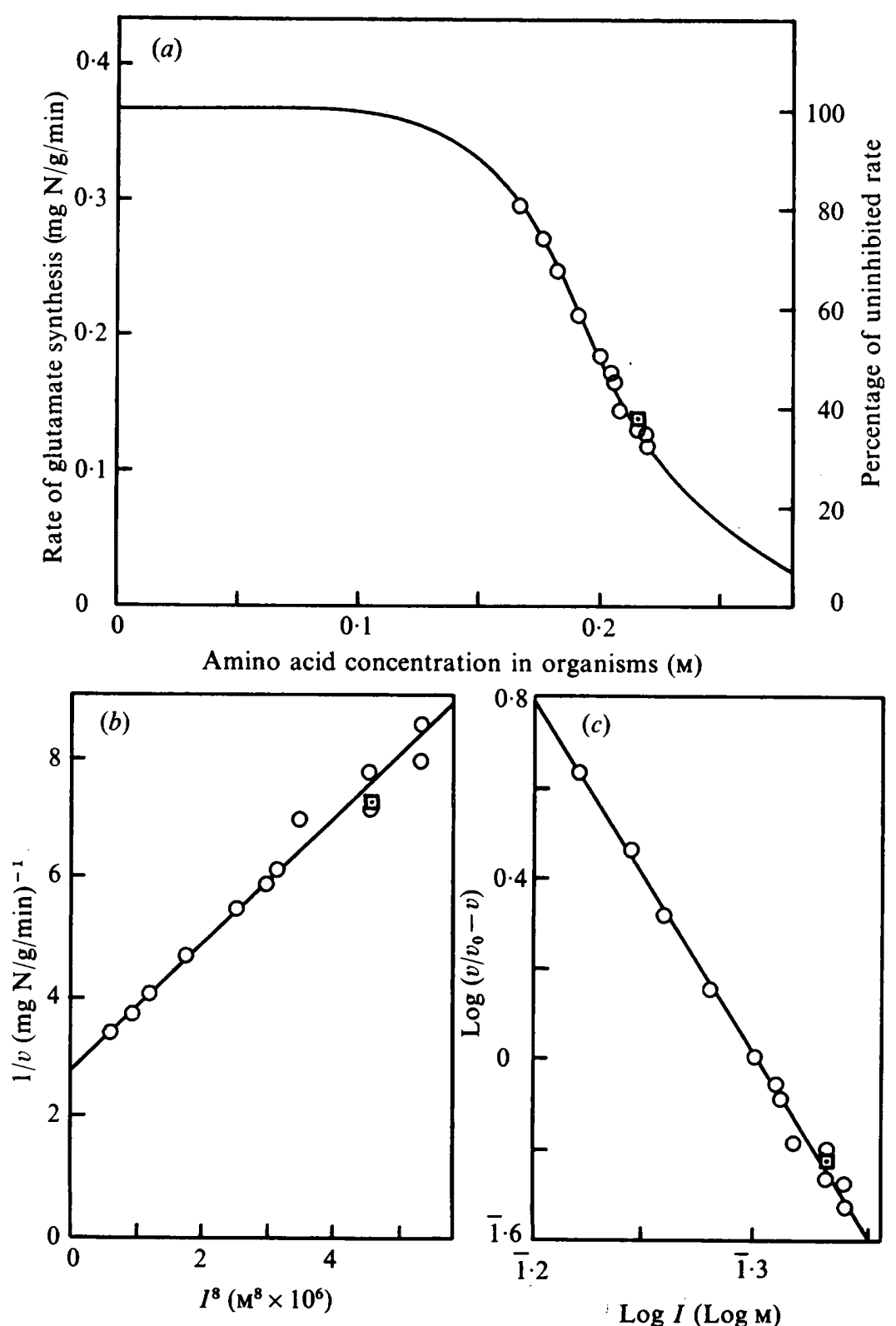

Fig. 3. Kinetic analysis of the effect of amino acid pool concentration on the rate of synthesis of glutamic acid in yeast at $15{ }^{\circ} \mathrm{C}$. $\bigcirc$, Data derived from the nitrogen starvation experiments shown in Fig. 2; $\square$, steady-state value derived from Table I. (a) Relation between rate of synthesis and pool concentration. The theoretical curve is calculated from the best fit line in Fig. $3 c$. $(b)$ Plot of reciprocal of rate of synthesis against eighth power of amino acid concentration $I$. (c) Hill plot. The slope gives a Hill number of $8 \cdot 0$.

constant, the total level of $\alpha$-amino nitrogen affords a reasonably good measure of the effective concentration of inhibitory ligands.

Studies in our laboratory (Jones, I968) on the inhibitory effects in vitro of both'individual amino compounds and a full mixture corresponding to the yeast amino acid pool revealed 
complex co-operative kinetics with Hill numbers varying between 2 and 6 . Repeated freezing and thawing of the enzyme brought about a partial desensitization to inhibition and resulted in lower Hill numbers, so an allosteric inhibition of the enzyme is clearly indicated.

The normal response of the enzyme in vitro to varying amino acid level was only qualitatively similar to the in vivo data described here. The response in vivo was apparently far sharper and we therefore attempted to quantify this by the analysis presented in Fig. 3. The experimental data covered only a restricted range of rates and amino acid concentrations and any further analysis had to depend on adequate extrapolation. A plot of the reciprocal of rate against amino acid concentration was suggested by analogy with classical enzymeinhibitor response, but this graph was extremely curvilinear. We therefore plotted against various powers of inhibitor concentration and with the eighth power fitted the data with a straight line (Fig. $3 b$ ). Extrapolation of this line yielded an estimate of the uninhibited rate of glutamate synthesis, and this value was used to construct a Hill plot (Fig. $3 c$ ).

The reciprocal power plot and the Hill plot are effectively equivalent tests for the degree of co-operativity, so it is not surprising that the Hill plot should have a slope of $8 \cdot 0$. This number is appreciably larger than that derived from the in vitro results, but there are indications that the Hill number in vitro varies with the concentration of the enzyme so that the enzyme in vivo may be more highly aggregated than when diluted by extraction from the organism and in enzyme assay. Certainly there is circumstantial evidence from the experiments of Jones (1968) that the lower the uninhibited rate of an enzyme preparation the smaller its Hill number. Appropriate extrapolation of these data suggests that at very high enzyme concentrations the Hill number would approach 8 or 9. Such large Hill numbers imply the existence of a highly oligomeric protein. Structural studies on the enzyme from yeast (Jones, 1968; A. P. Sims \& B. F. Folkes, unpublished) show the commonest form in vitro to be a hexamer of approximately 270000 daltons $\left(s_{20, \mathrm{w}}=\mathrm{II} \cdot 4\right)$, the monomer being 44000 daltons; these values compare closely with those for the enzyme from Neurospora (Wootton, Taylor \& Fincham, 1972; Gore, 1972; Blumenthal \& Smith, 1973). Treatment of the yeast enzyme with sodium dodecyl sulphate or urea yields an inactive sub-unit of 22000 daltons. Fincham \& Coddington (1963) reported a similar observation for Neurospora, urea treatment giving a protein with a $s_{20, \mathrm{w}}$ of 2.5 ; we estimate this to indicate a molecular weight of about 22500 . Gel filtration of purified yeast enzyme in the presence of $0.2 \mathrm{M}$-arginine revealed that an appreciable proportion of the protein was a 530000 dalton aggregate (Jones, 1968); this represents a dodecamer for which a Hill number of 8 to 9 would not be improbable. The in vivo data suggest that under cellular conditions this is the predominant form.

\section{Transient effects on the rate of glutamate synthesis following a shift in temperature}

An alternative way of disturbing the steady-state situation is by subjecting the yeast culture to a sudden shift in temperature (Fig. 4). The culture was grown in steady state at I $5{ }^{\circ} \mathrm{C}$ with a residual ammonia level of about $5 \mu \mathrm{g} \mathrm{N} / \mathrm{ml}$ and the experiment was initiated by switching from the original inflow medium to one where the ammonia was labelled with $30 \%$ atom excess ${ }^{15} \mathrm{~N}$. Five minutes after this switch, when the isotope level within the turbidostat had built up sufficiently to allow accurate estimates of glutamate synthesis to be made, the culture temperature was raised as rapidly as possible by the addition of hot water to the external water bath. The turbidostat temperature reached $21 \cdot 5{ }^{\circ} \mathrm{C}$ about $5 \mathrm{~min}$ after beginning heating and was then maintained at this level.

The rise in temperature was followed almost immediately by an increased rate of glutamate synthesis and a fall in the amino acid pool, apparently resulting from an increase in the rate 


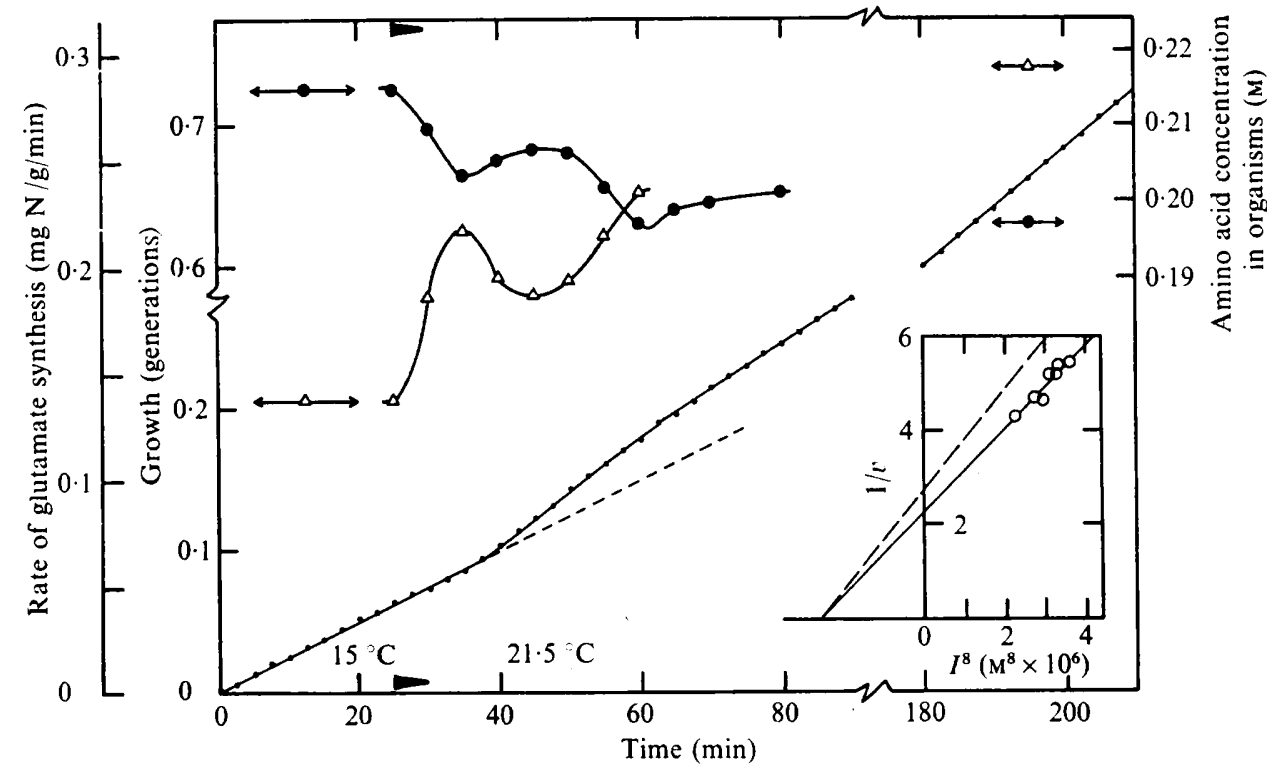

Fig. 4. The effect of a transition in temperature from 15 to $21.5{ }^{\circ} \mathrm{C}$ on the rate of glutamate synthesis. Steady-state yeast in a turbidostat at $15{ }^{\circ} \mathrm{C}$ was switched to a ${ }^{15} \mathrm{~N}$-labelled inflow and then raised to $2 \mathrm{I} \cdot 5^{\circ} \mathrm{C}$ (see text for details). $\triangle$, Rate of glutamate synthesis; $\leftarrow \triangle \rightarrow$, steady-state rate of glutamate synthesis at 15 and $21 \cdot 5{ }^{\circ} \mathrm{C}$ (the $21 \cdot 5{ }^{\circ} \mathrm{C}$ value is estimated from the rate of total nitrogen assimilation assuming $75 \%$ to be into glutamate); 0 , concentration of the total free amino acid pool ; $\leftarrow \rightarrow$, steady-state pool concentrations at 15 and $2 \mathrm{I} \cdot 5^{\circ} \mathrm{C}$. The lower graph represents the turbidostat input or growth trace (cf. the legend to Fig. 2). Inset: A plot of the reciprocal of rate of glutamate synthesis against the eighth power of amino acid concentration $(O)$. Only those data relating to $2 \mathrm{I} \cdot 5^{\circ} \mathrm{C}$ have been used. For comparison the corresponding graph for $15{ }^{\circ} \mathrm{C}$ (from Fig. $3 \mathrm{~b}$ ) is also shown (broken line). The solid line represents the best fit to the $2 \mathrm{I} \cdot 5^{\circ} \mathrm{C}$ points for a line passing through the 'apparent $K_{i}^{\prime}$ ' derived by the extrapolation of the $15{ }^{\circ} \mathrm{C}$ data.

of amino acid utilization which exceeded the increased rate of synthesis. Shortly afterwards the amino acid pool again rose and oscillated towards its final steady-state value at the new temperature. Glutamate synthesis again showed a mirror-image correspondence with the pool data, so that after its initial rise the glutamate rate curve fell sharply before rising again in concert with the next drop in amino acid level. As with the nitrogen starvation experiments, the growth rate of the organisms apparently played no direct part in the control of these phenomena. In this case the dilution rate of the culture remained constant for more than I 5 min after the temperature shift; in a characteristic way it then increased rapidly for a time before falling back to a rate intermediate between the other two.

Although the data in this experiment are restricted, we have attempted a brief analysis. Again, a plot of reciprocal of rate against the eighth power of amino acid concentration can be well fitted by a straight line (see inset to Fig. 4). Since in this experiment the temperature was higher than that relating to the data in Fig. 3, the estimated maximum velocity was appreciably higher, but a relatively good fit to the data could be made by assuming that the effective affinity of the enzyme for its inhibitory ligands was unchanged. The best fit regression line calculated by appropriately weighted least squares did not extrapolate to the same 'apparent $K_{i}$ ' value as the data in Fig. 3, but the two values were not significantly different. This suggested the same basic feedback mechanism, the only difference in the two sets of data being the effect of temperature on rate. However, the increase in $V_{\max }$ is far smaller 


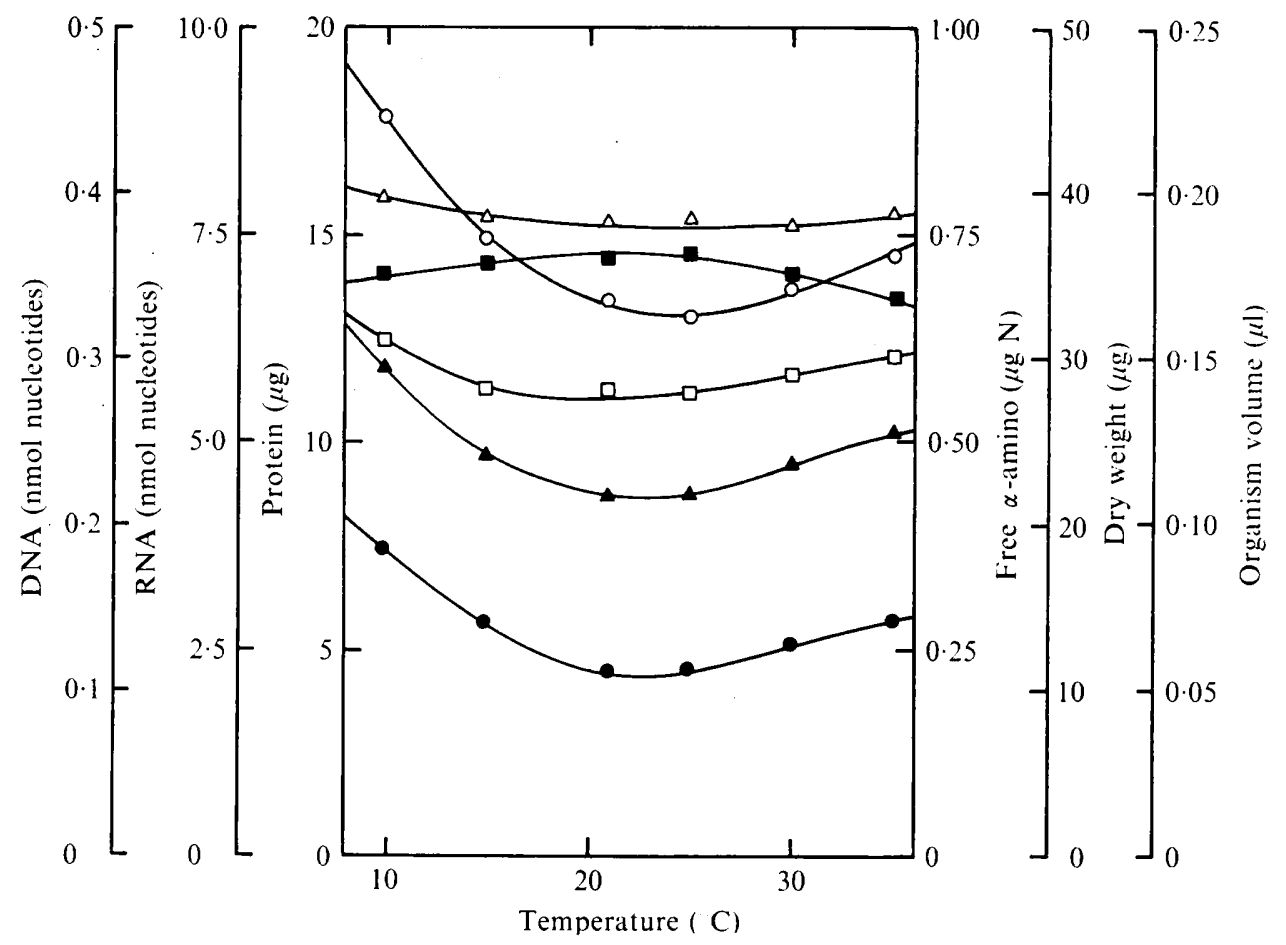

Fig. 5. Effect of culture temperature on the size and composition of yeast cells grown in steady state in a turbidostat. All results are expressed per $10^{6}$ cells. $O$, Dry weight; $\square$, total protein; $\triangle$, DNA; $\boldsymbol{\square}, \mathrm{RNA} ; \boldsymbol{\theta}$, total $\alpha$-amino nitrogen; $\boldsymbol{\Delta}$, mean cell volume.

than might be expected; the ratio for $2 \mathrm{I} \cdot 5{ }^{\circ} \mathrm{C} / 15^{\circ} \mathrm{C}$ is little more than $\mathrm{I} \cdot 2$, whereas studies on the isolated enzyme in vitro indicate a ratio of $\mathrm{I} \cdot 45$ (see Fig. $6 \mathrm{~b}$ ). Apparently therefore some other, more general, restraint on enzyme activity must have been operating under the conditions of this experiment, e.g. restrictions in the supply of $\alpha$-oxoglutarate and/or NADPH occasioned by a failure of carbohydrate catabolism to keep pace with the increased needs following upon an increase in temperature. Perhaps additional synthesis of appropriate enzymes is necessary before these needs can be fully met. It is clear that appropriate metabolic adjustments are eventually made, because by one generation after the start of the experiments the growth rate had markedly increased above its previous steady rate and the amino acid pool had attained its steady-state value (Fig. 4). Although glutamate synthesis was not measured at this time, it may be assumed that it too had reached its steady-state condition.

\section{Regulation of glutamate synthesis under steady-state conditions at different temperatures}

The experimental results described in the last section suggest that the feedback response of glutamate dehydrogenase to varying amino acid levels may be relatively unaffected by changes in temperature, the only effect being on the basic level of enzymic activity before the application of feedback restraint. Unlike simple enzymic reactions, complex biological processes such as growth or respiration do not exhibit a simple response to temperature consistent with a single constant heat of activation (see, for example, Forward, 1960). Consequently, for any enzymic reaction which is a component of such complex processes, 

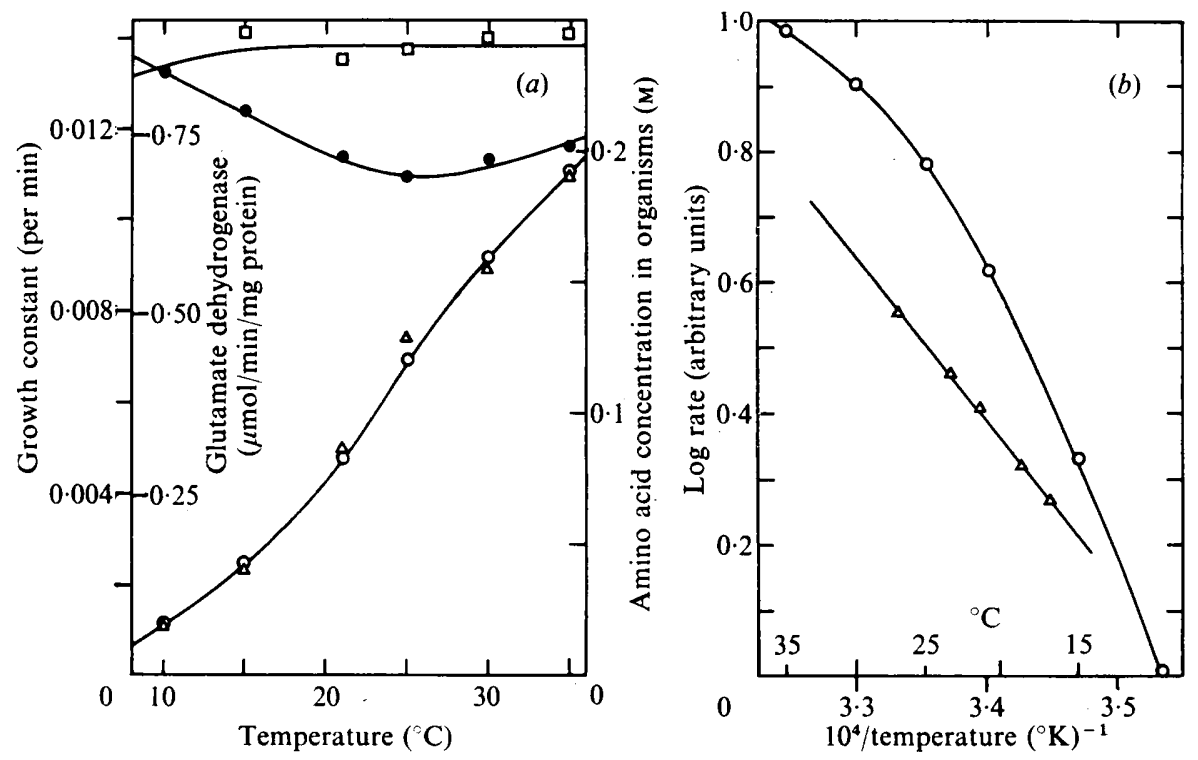

Fig. 6. A test of the ability of the amino acid-mediated feedback response of glutamate dehydrogenase to regulate glutamate synthesis to match the growth requirement at different temperatures. (a) $\bigcirc$, Growth constant; $\odot$, concentration of the total free amino acid pool; $\square$, specific activity of NADP-glutamate dehydrogenase/unit of cellular protein; $\triangle$, relative rates of glutamate synthesis calculated from the enzyme concentration and corrected for feedback inhibition and the effect of temperature (the $10{ }^{\circ} \mathrm{C}$ point has been set to coincide with the growth constant at that temperature). (b) Arrhenius plots of the effect of temperature on the yeast growth constant $(O)$ and the in vitro rate of NADP-glutamate dehydrogenase $(\triangle)$.

there must be some degree of regulatory control to modify the simple temperature response of the individual enzyme to match the needs of the total process which will be varying in a far more complex way. We therefore examined how far this additional control could, in the case of glutamate dehydrogenase, be attributed solely to the operation of the amino acid mediated feedback response.

Yeast was grown under a wide range of temperatures at turbidity settings which ensured that the effective concentration of ammonia in the fermentor vessel was between 4 and $6 \mu \mathrm{g}$ $\mathrm{N} / \mathrm{ml}$. When, at each temperature, the yeast came into steady state, measurements of growth rate were made and samples were taken for chemical analysis and enzyme assay. Cell size was also measured. The results are summarized in Figs. 5 and 6.

The organisms became smaller as the centre of the temperature range was approached (Fig. 5), not only with respect to organism volume and dry weight but also protein content. The DNA content per organism changed little over the temperature range, the slight increases at both very high and very low temperatures probably merely reflecting changes in the proportion of organisms in which DNA replication had not yet been followed by cell division. The RNA level fluctuated more widely, increasing markedly towards the centre of the growth range. Viewed against the general trend for decreasing cell size at these temperatures, this resulted in especially large increases in the ratio of RNA to protein, reaching a maximum at about $25^{\circ} \mathrm{C}$.

The quantity of free amino acids per cell decreases even more than the majority of cell components as the cell volume falls, so the amino acid concentration fell and reached a minimum at about $25^{\circ} \mathrm{C}$ (Fig. 6a). This is the temperature at which there is the greatest 


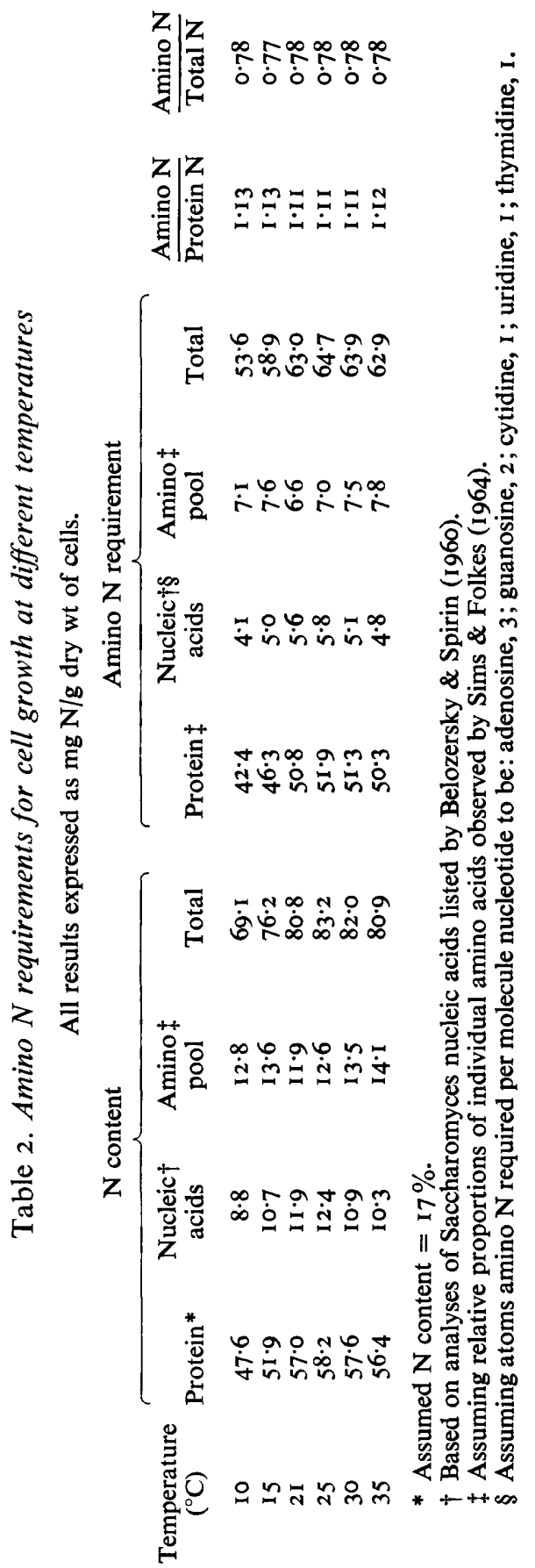


difference between the relative effects of temperature on yeast growth and on a simple enzymic reaction. This can be seen most clearly in Arrhenius plots of yeast growth and of the in vitro rate of glutamate synthesis by NADP-linked glutamate dehydrogenase (Fig. $6 b$ ), which indicate that their ratio is maximal at about $25^{\circ} \mathrm{C}$. This means that if, within the yeast cell, the glutamate dehydrogenase is to assimilate nitrogen at a rate sufficient to maintain growth, it will have to be under far less feedback restraint at $25^{\circ} \mathrm{C}$ than it is at either 10 or $35^{\circ} \mathrm{C}$. The observed changes in amino acid pool concentration were qualitatively consistent with these conclusions.

However, to test whether the feedback control was sufficient in this case to match enzymic rate to growth requirement, it was necessary to examine the data quantitatively. If the growth requirement is to be met, a unit weight of yeast protein must have the enzymic capability of synthesizing, during one generation, the equivalent of all the amino acids utilized in the synthesis of itself and of the other compounds, such as nucleic acids, associated with it in the living organism. For simplicity we assumed that this total amino acid requirement per generation per unit of protein is constant, irrespective of culture temperature; this assumption is correct to within a few per cent (Table 2). We could then calculate in relative terms the potential amino acid synthesis per generation at each temperature, if we knew the glutamate dehydrogenase level in the yeast (expressed as units of enzyme per unit weight of protein), the percentage of feedback inhibition due to the amino acid pool and the temperature coefficient of the enzyme in vitro. The specific activity per unit of protein was almost constant over the temperature range (Fig. $6 a$ ), the percentage feedback inhibition at various amino acid concentrations could be deduced from the graph in Fig. $3 a$, and the heat of activation of the isolated enzyme was approximately $47900 \mathrm{~J} / \mathrm{mol} /{ }^{\circ} \mathrm{C}$ (Fig. $6 b$ ). Calculations indicated that the potential enzymic capacity, after correction for feedback inhibition and the effect of temperature, was between four and five times greater than the growth requirement, suggesting that the enzyme in vivo was likely to have been under some degree of substrate limitation. However, if allowance was made for these effects by matching the Io ${ }^{\circ} \mathrm{C}$ estimate to the observed growth rate, the corrected synthetic capacities for the other temperatures fell on to the temperature-growth curve (Fig. $6 a$ ). The close correspondence revealed here makes it highly probable that the feedback effect of the amino acid pool is alone responsible for the regulation of glutamate dehydrogenase activity to meet the changing requirements of yeast cells in response to different temperatures.

\section{DISCUSSION}

Our experimental results offer clear indications that a major control governing the synthesis of glutamic acid in yeast is a feedback inhibition mediated by the organism's total pool of amino acids. Since, when yeast is grown on an inorganic source of nitrogen, glutamic acid is the precursor of all the $\alpha$-amino $N$ of other amino acids, a type of end-product inhibition is involved. The situation is unusual in the lack of specificity of the feedback response, although the biological relevance of this is immediately apparent. Mechanisms for the interconversion of amino acids in yeast are very flexible and almost any amino acid can act, through transamination or other processes, as the source of nitrogen for the synthesis of any other. In such a situation it makes good sense to have a system which is relatively unselective in its feedback response to amino nitrogen, in the mechanism controlling the rate of de novo amino synthesis. The ability of yeasts and other micro-organisms to 'spare' the synthesis of new amino groups, when any amino acid is already available as a nitrogen source, clearly resides in this wide response of the regulatory system. 
In assessing the quantitative validity of the results, we must admit the naivety of our experimental approach, since we have ignored differences in amino acid distribution between cytoplasm and vacuole, and variations of pool composition and enzyme level in cells of different ages (cf. Wiemken \& Nurse, 1973). These complexities make it impossible to deduce from the in vivo data enzyme kinetic parameters such as the dissociation constants of the enzyme-inhibitor complexes. At a less sophisticated level, however, our results offer, at least in relative terms, a generalized description of a physiological response. Certainly the strongest justification for the quantitative relationship between rate and ligand concentration revealed in these experiments is that it is apparently maintained under such a wide variety of experimental conditions and approaches, including nitrogen starvation, temperature transition, and even the regulation of steady-state assimilation at different temperatures.

Perhaps the most striking feature of the response curve is the sharpness of the cut-off in glutamate synthesis as a critical amino acid concentration is approached. The large Hill number generated here indicates that considerable complexity must underlie this phenomenon and suggests that the evolution of such an enzyme structure has brought appreciable selective advantage. Presumably this must be concerned with the need not to waste energy in unnecessary amino synthesis if alternative exogenous sources are available, while at the same time maintaining relatively high concentrations of amino acids within the cell so as to allow protein synthesis to continue at maximum rate. This is made possible by the system of control we have observed.

However, experimental data presented here reveal that this feedback response is not the only factor controlling the rate of glutamate synthesis in yeast; there are indications that the availability of carbon precursors or of ammonia may limit the process of assimilation. Ammonia is particularly important and at low concentrations the steady-state amino acid levels are markedly reduced; since this leads to a corresponding reduction in the feedback restraint on the enzyme, the absolute rates of nitrogen uptake are affected less than the size of the amino acid pool would suggest. An extreme example of the effect of decreased ammonia availability arises when nitrate is the source of nitrogen for growth. Then the endogenous ammonia level is so low that the amino pool is decreased to a point at which virtually all feedback restraint upon the glutamate dehydrogenase is removed (Sims et al. 1968). The enzyme itself is also considerably derepressed, in marked contrast to the extremely constant enzyme levels observed in the present experiments.

One further comment concerns the relative contributions to the total uptake of nitrogen made by the various pathways of nitrogen assimilation. Under steady-state conditions about $75 \%$ of the total nitrogen is assimilated into the amino group of glutamic acid; since this figure is based on determinations made at several different temperatures, and also agrees closely with the approximate calculations of amino requirements made in Table 2, it must be a fairly accurate measure of the contribution made by glutamate dehydrogenase activity to the total nitrogen assimilation. Our data offer only indirect estimates of the rate of glutamine synthesis based on ${ }^{15} \mathrm{~N}$ incorporation into glutamine-amino, and unfortunately the data of Sims \& Ferguson (1974) on amide labelling were derived from experiments carried out at significantly higher ammonia levels. Both sets of data, however, indicate that 12 to $15 \%$ of the assimilated nitrogen probably passes through the amide of the glutamine pool, a value considerably lower than might be expected in view of the evidence offered by Lacroute, Piérard, Grenson \& Wiame (1965) that in Saccharomyces cerevisiae glutamine amide is used in the synthesis of carbamyl phosphate. Our data are consistent with either the direct assimilation of ammonia into carbamyl phosphate such as apparently occurs in Neurospora crassa (Davis, 1965), or alternatively with the use of newly synthesized glutamine amide prior to 
its entry into the glutamine pool. We can only hypothesize that the remaining to to $12 \%$ of assimilation must pass via one or other of these means into carbamyl phosphate. There is little doubt however as to the overall processes of nitrogen assimilation in Candida, and of these it is clear that the synthesis of glutamic acid is quantitatively by far the most important.

We are indebted to the Science Research Council for financial support through grants B/SR/I29I and B/SR/5II6. About half of this work was carried out in the Department of Botany, University of Bristol, and we are grateful to Professor E. W. Yemm for support and facilities. A major part of the original design and development of the turbidostat is due to $\mathrm{Mr} \mathrm{B}$. Wurzberger, who also gave technical support in the Bristol experiments. Others involved in occasional experiments in Bristol were Dr A. H. Bussey and Dr P. E. Stanley (who also assisted in the construction of the first turbidostat). At Norwich, turbidostat design has been furthered by Mr P. E. Le Fevre, Mr A. J. D. Cooke and Mr C. J. Wright. Dr A. R. Ferguson and Mr S. G. Howitt assisted in one experiment. Mr D. Walls has given skilled technical help and prepared the Figures.

\section{REFERENCES}

Belozersky, A. N. \& Spirin, A. S. (1960). Chemistry of the nucleic acids of micro-organisms. In The Nucleic Acids, vol. 3, pp. I47-I 85. Edited by E. Chargaff and J. N. Davidson. New York and London: Academic Press.

Blumenthal, K. M. \& Smith, E. L. (1973). Nicotinamide adenine dinucleotide phosphate-specific glutamate dehydrogenase of Neurospora. I. Isolation, sub units, amino acid composition, sulphydryl groups and identification of a lysine residue reactive with pyridoxal phosphate and $\mathrm{N}$-ethylmaleimide. Journal of Biological Chemistry 248, 6002-6008.

Casselton, P. J. (1969). Concurrent regulation of two enzymes in fungi. Science Progress 57, 207-227.

DAvis, R. H. (1965). Carbamyl phosphate synthesis in Neurospora crassa. I. Preliminary characterization of arginine-specific carbamyl phosphokinase. Biochimica et biophysica acta 107, 44-53.

Ferguson, A. R. \& Sims, A.P. (I97I). Inactivation in vivo of glutamine synthesis and NAD-specific glutamate dehydrogenase: its role in the regulation of glutamine synthesis in yeasts. Journal of General Microbiology 69, 423-427.

Ferguson, A. R. \& Sims, A. P. (1974). The regulation of glutamine metabolism in Candida utilis: the role of glutamine in the control of glutamine synthetase. Journal of General Microbiology 80, I59-1 71.

Fincham, J. \& CoDdington, A. (I963). The mechanism of complementation between am mutants of Neurospora crassa. Cold Spring Harbor Symposia on Quantitative Biology 28, 51 7-527.

ForWARD, D. F. (1960). Effect of temperature on respiration. In Encyclopedia of Plant Physiology, vol. 12, part 2, pp. 234-258. Edited by W. Ruhland. Berlin, Göttingen and Heidelberg: Springer-Verlag.

Gore, M. (1972). Studies of the glutamate dehydrogenase of Neurospora crassa. Ph.D. Thesis, University of East Anglia.

JoNes, R. B. K. (1968). Glutamate dehydrogenase in Torulopsis utilis. M.Sc. Thesis, University of Bristol.

Lacroute, F., Piérard, A., Grenson, M. \& Wiame, J. M. (I965). The biosynthesis of carbamyl phosphate in Saccharomyces cerevisiae. Journal of General Microbiology 40, I 27-142.

Lowry, O. H., Rosebrough, N. J., Farr, A. L. \& Randall, R. J. (I95I). Protein measurement with the Folin phenol reagent. Journal of Biological Chemistry 193, 265-275.

Shapiro, B. M. \& StadtMan, E. R. (1970). The regulation of glutamine synthesis in micro-organisms. Annual Reviews of Microbiology 24, 50I-524.

Sims, A. P. \& Ferguson, A. R. (1974). The regulation of glutamine metabolism in Candida utilis: studies with ${ }^{15} \mathrm{NH}_{3}$ to measure in vivo rates of glutamine synthesis. Journal of General Microbiology 80, I43-I 58 .

Sims, A. P. \& FolKes, B. F. (1964). A kinetic study of the assimilation of ${ }^{15} \mathrm{~N}$-ammonia and the synthesis of amino acids in an exponentially growing culture of Candida utilis. Proceedings of the Royal Society B 159, 479-502.

Sims, A. P., Folkes, B. F. \& Bussey, A. H. (1968). Mechanisms involved in the regulation of nitrogen assimilation in micro-organisms and plants. In Recent Aspects of Nitrogen Metabolism in Plants (First Long Ashton Symposium, 1967), pp. 9I-I I4. Edited by E. J. Hewitt and C. V. Cutting. London and New York: Academic Press. 
Weatherburn, M. W. (1967). Phenol hypochlorite reaction for determination of ammonia. Analytical Chemistry 39, 97I-974.

West, D. J., Tuveson, R. W., Barratt, R. W. \& Fincham, J. R. S. (1967). Allosteric effects in nicotinamide adenine dinucleotide phosphate-specific glutamate dehydrogenase from Neurospora. Journal of Biological Chemistry 242, 2 I 34-2 I 38.

Wiemken, A. \& Nurse, P. (I973). Isolation and characterization of the amino-acid pools located within the cytoplasm and vacuoles of Candida utilis. Planta 109, 293-306.

Wootton, J. C., TAYlor, J.G. \& FInChaM, J. R. S. (I972). The subunit structure of the nicotinamide-adenine dinucleotide phosphate-dependant glutamate dehydrogenase of Neurospora crassa. Biochemical Journal 127, 3 I $P$.

YEMM, E. W. \& Folkes, B. F. (I954). The regulation of respiration during the assimilation of nitrogen in Torulopsis utilis. Biochemical Journal 57, 459-508. 